\title{
OPEN An increasing role of pyrethroid-resistant Anopheles funestus in malaria transmission in the Lake Zone, Tanzania
}

\author{
Nancy S. Matowo ${ }^{1 凶}$, Jackline Martin ${ }^{2,4}$, Manisha A. Kulkarni ${ }^{3}$, Jacklin F. Mosha², \\ Eliud Lukole ${ }^{1,2}$, Gladness Isaya ${ }^{4}$, Boniface Shirima ${ }^{4}$, Robert Kaaya ${ }^{4}$, Catherine Moyes ${ }^{5}$, \\ Penelope A. Hancock ${ }^{5}$, Mark Rowland ${ }^{1}$, Alphaxard Manjurano ${ }^{2}$, Franklin W. Mosha ${ }^{4}$, \\ Natacha Protopopoff ${ }^{1,6}$ \& Louisa A. Messenger ${ }^{1,6 \bowtie}$
}

Anopheles funestus is playing an increasing role in malaria transmission in parts of sub-Saharan Africa, where An. gambiae s.s. has been effectively controlled by long-lasting insecticidal nets. We investigated vector population bionomics, insecticide resistance and malaria transmission dynamics in 86 study clusters in North-West Tanzania. An. funestus s.l. represented $94.5 \%(4740 / 5016)$ of all vectors and was responsible for the majority of malaria transmission $(96.5 \%)$, with a sporozoite rate of $3.4 \%$ and average monthly entomological inoculation rate (EIR) of 4.57 per house. Micro-geographical heterogeneity in species composition, abundance and transmission was observed across the study district in relation to key ecological differences between northern and southern clusters, with significantly higher densities, proportions and EIR of An. funestus s.l. collected from the South. An. gambiae s.I. (5.5\%) density, principally An. arabiensis (81.1\%) and An. gambiae s.s. (18.9\%), was much lower and closely correlated with seasonal rainfall. Both An. funestus s.l. and An. gambiae s.l. were similarly resistant to alpha-cypermethrin and permethrin. Overexpression of CYP9K1, CYP6P3, CYP6P4 and CYP6M2 and high L1014S-kdr mutation frequency were detected in An. gambiae s.s. populations. Study findings highlight the urgent need for novel vector control tools to tackle persistent malaria transmission in the Lake Region of Tanzania.

The widespread deployment of primary vector control interventions, principally long-lasting insecticidal nets (LLINs) and indoor residual spraying (IRS), has substantially reduced malaria incidence across sub-Saharan Africa $^{1,2}$. Between 2000 and 2015, 68\% of the 1.5 billion malaria cases averted can be attributed to LLINs alone ${ }^{1}$. However, current estimates indicate the rates of decline have begun to stagnate ${ }^{2}$. Tanzania is among the 10 subSaharan African countries where malaria burden is concentrated ${ }^{3}$, contributing to $5 \%$ of global malaria deaths ${ }^{2}$. Malaria infection varies nationwide with an average prevalence of $7.3 \%$ in children under 5 years of age in $2017^{4}$. Vector control by the National Malaria Control Programme (NMCP) is based on sustaining high LLIN access and use $^{5}$, via universal coverage campaigns supplemented with continuous distribution from school net programmes, antenatal care campaigns and the expanded programme for immunization; and targeted IRS in high transmission areas in the North-West ${ }^{6}$. Effective and sustainable malaria vector control is plagued by a number of challenges, including the evolution of vector behavioural and physiological resistance to current control interventions ${ }^{7}$. In the majority of sentinel districts across Tanzania, Anopheles mosquitoes have demonstrated reduced susceptibly to at least one public health insecticide ${ }^{8,9}$.

Continued use of insecticide-based malaria control tools has been linked with changes in Anopheles feeding and resting behaviors and relative species composition ${ }^{10-13}$. In some countries, Anopheles funestus sensu stricto (s.s.) has historically played a significant role in malaria transmission ${ }^{14-17}$ largely due to its predominantly

\footnotetext{
${ }^{1}$ Department of Disease Control, London School of Hygiene and Tropical Medicine, London, UK. ${ }^{2}$ National Institute for Medical Research, Mwanza Medical Research Centre, Mwanza, Tanzania. ${ }^{3}$ School of Epidemiology and Public Health, University of Ottawa, Ottawa, Canada. ${ }^{4}$ Kilimanjaro Christian Medical University College, Moshi, Tanzania. ${ }^{5}$ Big Data Institute, Li Ka Shing Centre for Health Information and Discovery, University of Oxford, Oxford, UK. ${ }^{6}$ These authors contributed equally: Natacha Protopopoff and Louisa A. Messenger. ${ }^{\square}$ email: Nancy.Matowo@Ishtm.ac.uk; louisa.messenger@Ishtm.ac.uk
} 

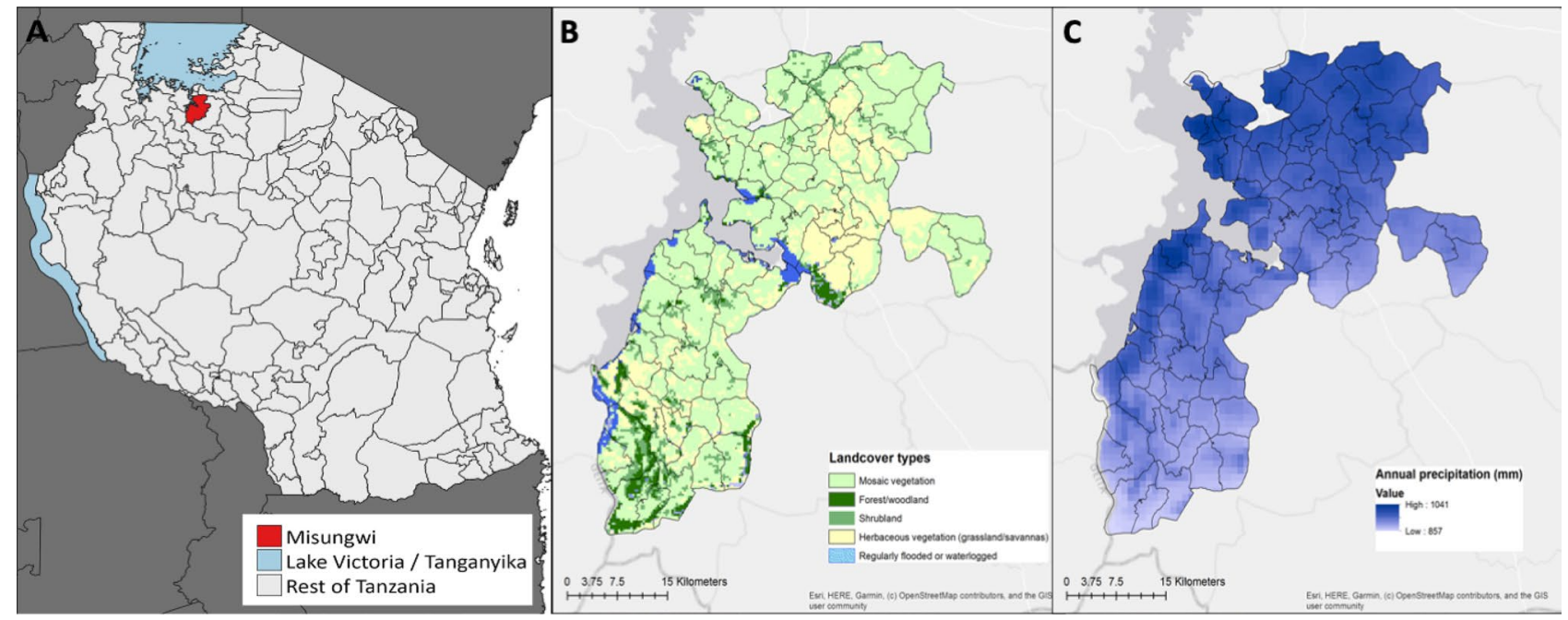

Figure 1. Study area in Misungwi district, North-West Tanzania, displaying (A) location of Misungwi in the Lake Region; (B) landcover features of study clusters; and (C) annual precipitation ( $\mathrm{mm}$ ) in study clusters. The maps were created using ArcGIS software 10.8.1 (Environmental Systems Research Institute (ESRI) Redlands, California, USA, http://www.esri.com/arcgis) $)^{32}$.

anthropophilic and endophilic tendencies ${ }^{18}$, intense pyrethroid resistance ${ }^{19-24}$ and greater daily survival probabilities (higher parity rates) $)^{25,26}$. In other areas, notably South-East Tanzania ${ }^{25,27}$, far North-West Tanzania ${ }^{28}$ and parts of Kenya ${ }^{13}$, this species is rapidly replacing An. gambiae s.s. and An. arabiensis, following the scale-up of vector control interventions, and has been found with some of the highest Plasmodium sporozoite rates ${ }^{25}$. Increasing An. funestus population densities and vectorial capacity in these regions may be due to recent escalations in pyrethroid resistance intensities ${ }^{13,25,27}$, but also changes in aquatic larval habitats which are more permissible for An. funestus breeding 29 .

Malaria prevalence around Lake Victoria remains amongst the highest in Tanzania ${ }^{30}$, despite high communitylevel coverage with LLINs, and periodic IRS campaigns ${ }^{6,28}$. Factors driving persistent malaria transmission in the region, including the relative importance of An. funestus sensu lato (s.l.) as a major vector species, are poorly characterised but warrant investigation for the design and strategic deployment of new vector control tools. We assessed vector population bionomics, malaria transmission dynamics, phenotypic insecticide resistance and target site and metabolic resistance mechanisms in 86 study clusters in Misungwi district, North-West Tanzania, prior to a randomised controlled trial (RCT) assessing the efficacy of next-generation LLINs to improve malaria control $^{31}$.

\section{Results}

Household characteristics. A total of 1593 households were visited during two cross-sectional entomological field surveys, across 86 clusters in Misungwi district, North-West Tanzania on the southern shore of Lake Victoria, between August and December 2018 (Fig. 1A).

The district spans two agro-ecological zones, based on vegetation land cover and rainfall (Fig. 1), that are divided roughly into northern and southern clusters.

The study had an overall response rate of $86.2 \%$ (1372/1592), with consent to participate in the survey given from an adult/head of the household. Ten per cent (164) of dwellings were found vacant, $1.0 \%$ (16) were not located, $0.2 \%$ (3) not visited due to accessibility and $0.1 \%$ (2) were ineligible (no children under 15 years) during the survey period. A small proportion $2.2 \%$ (35) refused to participate in the study. The average altitude of study households was $1194.9 \mathrm{~m}$ above sea level (Table 1). Similar proportions of houses were classified as improved or unimproved, based on construction with modern or traditional materials, respectively (Table 1; Fig. 3A). Notably, few houses had mosquito proofing materials over the windows $(29.9 \%)$ and almost no houses had ceilings (97.2\%); 40.6\% of houses had open eaves (Table 1; Fig. 3A,B). The average household size was 6.6 persons and mean number of room/sleeping place was 2.7 per house. Forty-four per cent of households owned at least one livestock (mostly goats and cattle), which were usually kept outdoors about $20 \mathrm{~m}$ away from the house.

LLIN ownership was very high in the study area with the majority of families owning at least one LLIN (94.9\%); LLIN access was comparatively lower, however, the majority of households had enough LLINs to cover all of their sleeping places (62.3\%). About $54.2 \%$ of households were sprayed during the 2015 IRS National Malaria Control Campaign (Table 1). There were no significant differences in household characteristics, including size, altitude, construction materials, however population access to insecticide-treated net (ITN) access was slightly higher in northern than southern clusters (Table 1).

Vector distribution, species composition, relative abundance and seasonality. A total of 23,081 mosquitoes, comprising 23.1\% (5329) Anophelines and 76.9\% (17,752) Culicines, were collected using Centers for Disease Control and Prevention light traps (CDC-LTs) during two cross-sectional survey rounds between 


\begin{tabular}{|c|c|c|c|c|c|c|}
\hline \multirow[b]{2}{*}{ Outcome } & \multicolumn{2}{|l|}{ All } & \multicolumn{2}{|c|}{ Northern villages } & \multicolumn{2}{|c|}{ Southern villages } \\
\hline & $\mathbf{N}$ & $\%$ /mean $[95 \% \mathrm{CI}]$ & $\mathbf{N}$ & $\% /$ mean $[95 \% \mathrm{CI}]$ & $\mathbf{N}$ & $\% /$ mean $[95 \% \mathrm{CI}]$ \\
\hline Total HHs visited & 1592 & N/A & 980 & $\mathrm{~N} / \mathrm{A}$ & 613 & N/A \\
\hline Total consents given & 1373 & N/A & 847 & N/A & 526 & N/A \\
\hline Total HHs analysed & 1372 & N/A & 846 & $\mathrm{~N} / \mathrm{A}$ & 526 & N/A \\
\hline $\begin{array}{l}\text { Average number of people per } \\
\text { household }\end{array}$ & 1372 & $6.6[6.4-6.8]$ & 846 & $6.5[6.2-6.8]$ & 526 & $6.8[6.4-7.1]$ \\
\hline Average number of sleeping space & 1372 & $2.7[2.6-2.8]$ & 846 & $2.7[2.6-2.8]$ & 526 & $2.7[2.5-2.8]$ \\
\hline Average altitude (meters) & 1372 & $1194.9[1187.5-1202.4]$ & 846 & $1195.3[1186.2-1204.3]$ & 525 & $1194.4[1181.0-1207.8]$ \\
\hline$\%$ household with iron roof & 1372 & $67.4 \%[63.2-71.2]$ & 846 & $67.2 \%[61.0-72.7]$ & 526 & $67.7 \%[62.5-72.5]$ \\
\hline$\%$ household with open eaves & 1372 & $40.6 \%[37.0-44.3]$ & 846 & $38.4 \%[33.7-43.4]$ & 526 & $44.1 \%[38.6-49.8]$ \\
\hline$\%$ household with screened windows & 1372 & $29.9 \%[26.2-33.7]$ & 846 & $33.1 \%[28.1-38.5]$ & 526 & $24.5 \%[20.1-29.5]$ \\
\hline$\%$ houses made of brick walls & 1372 & $67.5 \%[62.5-72.1]$ & 846 & $68.9 \%[62.6-74.6]$ & 526 & $65.2 \%[56.6-72.9]$ \\
\hline$\%$ houses with no ceiling & 1372 & $97.2 \%[95.4-98.3]$ & 846 & $96.3 \%[93.5-98.0]$ & 526 & $98.5 \%[96.9-99.3]$ \\
\hline $\begin{array}{l}\% \text { houses with modern constructed } \\
\text { materials }\end{array}$ & 1372 & $51.8 \%[47.8-55.8]$ & 846 & $53.7 \%[48.1-59.1]$ & 526 & $48.9 \%[43.1-54.6]$ \\
\hline$\%$ household owning cattle and goats & 1372 & $43.8 \%[39.8-47.9]$ & 846 & $41.3 \%[36.0-46.8]$ & 526 & $47.7 \%[41.8-53.7]$ \\
\hline $\begin{array}{l}\% \text { of household owning at least one } \\
\text { ITN }\end{array}$ & 1372 & $94.9 \%[93.5-96.0]$ & 846 & $95.3 \%[93.5-96.6]$ & 526 & $94.3 \%[91.5-96.2]$ \\
\hline Mean number of ITN per house & 1372 & $2.3[2.2-2.4]$ & 846 & $2.4[2.2-2.5]$ & 526 & $2.1[2.0-2.3]$ \\
\hline $\begin{array}{l}\text { Population access to ITN (One net for } \\
\text { every two people) }\end{array}$ & 1372 & $67.9 \%[65.8-70.1]$ & 846 & $70.4 \%[67.8-73.1]$ & 526 & $63.9 \%[60.5-67.3]$ \\
\hline $\begin{array}{l}\% \text { HHs with enough nets to cover } \\
\text { their sleeping places }\end{array}$ & 1372 & $62.3 \%[59.0-65.7]$ & 846 & $64.1 \%[59.9-68.2]$ & 526 & $59.5 \%[53.7-65.4]$ \\
\hline$\%$ of household sprayed in 2015 & 1372 & $54.2 \%[49.8-58.5]$ & 445 & $52.5 \%[46.5-58.4]$ & 299 & $56.8 \%[50.3-63.2]$ \\
\hline
\end{tabular}

Table 1. Household characteristics in the study area in Northern and Southern clusters. The district spans two agro-ecological zones, based on vegetation land cover and rainfall (Fig. 1), that are divided roughly into northern and southern clusters. HH household, ITN insecticide-treated net, N/A not applicable.

\begin{tabular}{|l|l|l|l|l|l|l|}
\hline \multirow{2}{*}{ Outcome } & \multicolumn{2}{l}{ Overall } & \multicolumn{2}{l|}{ Northern villages } & \multicolumn{2}{l|}{ Southern villages } \\
\cline { 2 - 7 } & $\mathbf{N}$ & \%/ mean [95\% CI] & $\mathbf{N}$ & \%/mean [95\% CI] & N & \%/mean [95\% CI] \\
\hline Total HH/night collection & 1372 & N/A & 846 & N/A & 526 & N/A \\
\hline Mean mosquitoes per night per house & 23,081 & $16.8[11.6-22.0]$ & 9830 & $11.6[7.5-15.8]$ & 13,251 & $25.2[13.9-36.4]$ \\
\hline Mean Anopheles vectors & 5016 & $3.7[1.9-5.4]$ & 788 & $0.9[0.5-1.4]$ & 4228 & $8.0[3.9-12.1]$ \\
\hline Total An. gambiae s.l. & 276 & $0.2[0.1-0.3]$ & 176 & $0.2[0.1-0.3]$ & 100 & $0.2[0.1-0.3]$ \\
\hline Total An. funestus s.l. & 4740 & $3.5[1.7-5.2]$ & 612 & $0.7[0.3-1.1]$ & 4128 & $7.8[3.8-11.9]$ \\
\hline Total Culex species & 15,609 & $11.4[7.7-15.0]$ & 7417 & $8.8[5.6-12.0]$ & 8192 & $15.6[7.8-23.3]$ \\
\hline Proportion of An. arabiensis, n/N & $150 / 185$ & $81.1 \%[67.9-89.7]$ & $70 / 103$ & $68.0 \%[49.6-82.1]$ & $80 / 82$ & $97.6 \%[91.8-99.3]$ \\
\hline Proportion of An. gambiae s.s., n/N & $35 / 185$ & $18.9 \%[10.3-32.1]$ & $33 / 103$ & $32.0 \%[17.9-50.4]$ & $2 / 82$ & $2.4 \%[0.7-8.2]$ \\
\hline Proportion of An. funestus s.s., n/N & $710 / 764$ & $92.9 \%[89.7-95.2]$ & $202 / 220$ & $91.8 \%[85.9-95.4]$ & $508 / 544$ & $93.3 \%[89.1-96.0]$ \\
\hline Sporozoite rate, n/N & $67 / 1963$ & $3.4 \%[2.5-4.6]$ & $13 / 603$ & $2.2 \%[1.1-4.1]$ & $54 / 1360$ & $4.0 \%[2.9-5.4]$ \\
\hline Monthly EIR/house & 1349 & $4.4[1.2-7.7]$ & 831 & $0.6[0.1-1.2]$ & 518 & $9.6[2.7-16.4]$ \\
\hline
\end{tabular}

Table 2. Malaria vector species composition, sporozoite rate and entomological inoculation rate (EIR) per study zone. EIR entomological inoculation rate, $H H$ household, $N / A$ not applicable.

August and December 2018, for a total of 1372 trap nights (Fig. 3C). Most mosquito collections (82.1\%) did not experience rainfall and $35.6 \%$ of collections had moderate winds.

Of the Anophelines collected, 94.1\% (5016) were malaria vectors comprised of 94.5\% (4740) An. funestus s.l. and 5.5\% (276) An. gambiae s.l. Significantly greater numbers of An. funestus s.l. were collected across the study area compared to An. gambiae s.l., (average number of mosquitoes caught per trap per house per night were: An. gambiae s.l. = 0.20 [95\% CI 0.15-0.27], An. funestus s.l. = 3.45 [95\% CI 1.71-5.19]); p < 0.001) (Table 2). Within the An. funestus complex, the predominant species found was An. funestus s.s. $(92.9 \% ; 710 / 764$ selected for species-specific PCR); other species identified were An. parensis (6.5\%) and An. rivulorum (0.5\%). Of the 185 An. gambiae s.l. selected for sibling species identification, $81.1 \%$ were An. arabiensis and $18.9 \%$ were An. gambiae s.s. (Table 2).

Overall, significantly higher mosquito densities were observed in villages located in the southern clusters compared to the northern clusters (average number of Anopheles caught per trap per house per night in the 


\begin{tabular}{|l|l|l|}
\hline Outcome & Anopheles funestus s.l. & Anopheles gambiae s.l. \\
\hline Total collection nights & 1372 & 1372 \\
\hline Total number of mosquitoes collected & 4740 & 276 \\
\hline Mean collection in wet season & $2.87[1.09-4.65]$ & $0.23[0.11-0.36]$ \\
\hline Mean collection in dry season & $4.61[1.93-7.30]$ & $0.14[0.07-0.20]$ \\
\hline No. sample analysed for the presence of CSP & 1714 & 249 \\
\hline Number of sporozoite positive & 59 & 8 \\
\hline Overall SR \% (95\% CI) & $3.44 \%[2.52-4.68]$ & $3.21 \%[1.32-7.60]$ \\
\hline SR wet season & $3.82 \%[2.56-5.66]$ & $3.59 \%[1.34-9.23]$ \\
\hline SR dry season & $2.85 \%[1.71-4.72]$ & $1.85 \%[0.23-13.24]$ \\
\hline Mean monthly EIR (weighted) & $4.57[1.05-8.09]$ & $0.17[0.01-0.33]$ \\
\hline EIR wet season & $4.67[0.03-9.32]$ & $0.23[0.01-0.45]$ \\
\hline EIR dry season & $4.38[0.69-8.07]$ & $0.07[0.06-0.20]$ \\
\hline Estimate annual EIR (weighted) & $54.85[12.60-97.09]$ & $2.01[0.12-3.91]$ \\
\hline \% EIR contribution (weighted) & $96.47 \%$ & $3.53 \%$ \\
\hline
\end{tabular}

Table 3. Seasonal variation between An. funestus s.l. and An. gambiae s.l. sporozoite rate and entomological inoculation rate (EIR). CSP circumsporozoite protein, EIR entomological inoculation rate, SR sporozoite rate.

northern zone $=0.93$, southern zone $=8.04$; Density Ratio $(D R)=6.09[95 \%$ CI 3.00-12.38]; p $<0.001)$ (Table 2). There were significantly more An. funestus s.l. sampled from households in the southern part of the study area (average/house/night $=7.85)$, compared to the North $(0.72 ; \mathrm{DR}=7.92[95 \%$ CI 3.76-16.67]; $\mathrm{p}<0.001)$. However, there was no statistical difference in An. gambiae s.l. collected between the two locations with an average of 0.21 per night in the northern zone and 0.19 in the southern zone ( $\mathrm{DR}=1.28$ [95\% CI 0.69-2.38]; $\mathrm{p}=0.431)$ (Table 2).

Amongst sibling species of the An. gambiae complex, there were marked spatial and seasonal fluctuations. Most An. gambiae s.s. (94.3\%) were collected from the northern zone and more than $71.5 \%$ of An. funestus s.s. from the southern part. An. arabiensis was distributed throughout both study zones, but its abundance was slightly higher in the South (Table 2). Both An. funestus s.s. and An. arabiensis predominated throughout the study period, but An. gambiae s.s. abundance peaked in December in the middle of the rainy season. An analysis of bioclimatic and landcover characteristics across the study area demonstrated several ecological differences between the northern and southern zones, with the former composed mostly of grassland and cropland (91\%), with smaller proportions of shrubland and forest (7\%) and areas prone to regularly flooding (1\%); and the latter with less grassland and cropland (80\%) and greater proportions of shrubland and forest (14\%) and areas prone to regular flooding (3\%) (Supplementary table S1). Furthermore, differences in rainfall were also observed between the northern and southern zones, with villages in the North receiving slightly higher average annual precipitation $(959.5 \mathrm{~mm})$ than in the South $(911.5 \mathrm{~mm})$.

While overall An. gambiae s.l. density was low, it was closely correlated with seasonal rainfall patterns. Mean An. gambiae s.l. caught per house during the dry season (August and September; average precipitation of $5-7 \mathrm{~mm})$ was 0.14 but rose significantly by two-fold $(\mathrm{DR}=1.73$ [95\% CI $1.08-2.78$ ]; $\mathrm{p}=0.02)$ in the wet season (October, November and December; average precipitation of 147.3-158.5 mm). By comparison, significantly higher An. funestus s.l. densities were observed during the dry months [mean = 4.61; Incidence Rate Ratio $(\operatorname{IRR})=1.58, p=0.004]($ Table 3$)$.

The majority of sleeping spaces/beds where the CDC-LTs were installed had either Olyset $(60.5 \% ; 830 / 1372)$ or PermaNet 2.0 LLINs (36.0\%; 494/1372); 0.5\% (7) had both Olyset and PermaNet LLINs, previously distributed through mass universal replacement campaigns (URCs) that was conducted between 2014 and 2017 to achieve universal coverage ${ }^{33}$. The remaining $2.6 \%$ (35) of nets had no labels and $0.4 \%$ (6) were missing data on net type. There was no significant difference in malaria vector densities between rooms with the two main types of LLIN (average number of malaria vectors per house per night with Olyset LLINs $=3.59$ [95\% CI 1.64-.54], versus PermaNet 2.0 LLINs = 3.89 [95\% CI 1.78-6.00]; $\mathrm{p}=0.111$ ).

Plasmodium falciparum infection and entomological inoculation rate. A total of 1963 Anopheles mosquitoes (603 and 1360 from the northern and southern clusters, respectively) were tested for the presence of Plasmodium falciparum circumsporozoite protein (CSP), with 67 found infected, giving an overall sporozoite rate of 3.4\% [95\% CI 2.5-4.6] (Table 2). Of the An. gambiae s.l. and An. funestus s.l. individuals which tested CSP positive, $6.1 \%$ (4/66) were An. gambiae s.s., $1.5 \%$ (1/66) An. arabiensis, and 77.3\% (51/66) An. funestus s.s., respectively; the remaining samples could not be amplified by PCR. Sporozoite rates were similar in An. funestus s.l. (3.44\%) compared to An. gambiae s.l. (3.21\%) (Table 3).

Overall sporozoite rate varied across the study area with the highest rates observed in the southern zone (Table 2; odds ratio $(\mathrm{OR})=1.88$, [95\% CI 1.02-3.46]; $\mathrm{p}=0.044)$. In southern clusters, sporozoite rates for An. funestus s.l. was significantly higher than in northern clusters $(\mathrm{OR}=2.33$, [95\% CI 1.11-4.95]; $\mathrm{p}=0.028)$. The monthly sporozoite rate for An. funestus s.l. and An. gambiae s.l. fluctuated across the dry and wet seasons with slightly higher, but not significant, rates in the wet season (Table 3). An. funestus s.s. maintained malaria transmission across both seasons (sporozoite rates of 2.85\% [95\% CI 1.71-4.72] and 3.82\% [95\% CI 2.56-5.66], during the dry and wet seasons, respectively) while An. gambiae s.s. appeared to contribute to transmission mainly in 


\begin{tabular}{|l|l|l|l|l|}
\hline Outcome & CDC LT indoors & Furvela tent trap outdoors & Prokopack indoors & Prokopack outdoors \\
\hline Total HH/night collection & 96 & 96 & 96 & 96 \\
\hline Total Anopheles vectors & 536 & 464 & 56 & 52 \\
\hline Mean malaria vector & $5.6[2.3-8.8]$ & $4.8[2.9-6.7]$ & $0.6[0.2-0.9]$ & $0.5[0.1-1.0]$ \\
\hline Proportion An. gambiae s.l. & $33.6 \%[14.4-52.7]$ & $49.8 \%[32.2-67.4]$ & $51.8 \%[26.8-76.7]$ & $71.2 \%[43.0-99.3]$ \\
\hline Proportion An. funestus s.l. & $66.4 \%[47.3-85.6]$ & $50.2 \%[32.6-67.8]$ & $48.2 \%[23.3-73.2]$ & $28.8 \%[0.7-57.0]$ \\
\hline Proportion of An. arabiensis & $54.7 \%[25.5-81.0]$ & $51.8 \%[26.7-76.0]$ & $54.5 \%[12.5-91.0]$ & $89.5 \%[54.3-98.4]$ \\
\hline Proportion of An. gambiae s.s. & $45.3 \%[19.0-74.5]$ & $48.2 \%[24.0-73.3]$ & $45.5 \%[9.0-87.5]$ & $10.5 \%[1.6-45.7]$ \\
\hline Proportion of An. funestus s.s. & $96.4 \%[91.7-98.5]$ & $99.4 \%[95.4-99.9]$ & $100 \%$ & $100 \%$ \\
\hline Total Anopheles tested for CSP & 412 & 408 & 51 & 52 \\
\hline Number of sporozoite positive & 7 & 7 & 2 & 0 \\
\hline \% SR & $1.7 \%[0.8-3.5]$ & $1.7 \%[0.8-3.6]$ & $3.9 \%[1.0-14.4]$ & $0.0 \%$ \\
\hline
\end{tabular}

Table 4. Indoor and outdoor Anopheles feeding and resting behaviours and species composition. CDC Centers for Disease Control and Prevention, CSP circumsporozoite protein, $H H$ household, $L T$ light trap, SR sporozoite rate.

\begin{tabular}{|l|l|l|l|l|}
\hline Study ward & Location & Anopheles species tested & $\mathbf{3 0}$ min Knock-down [95\% CI] & $\mathbf{2 4}$ h Mortality [95\% CI] \\
\hline Ilujamate & Southern & An. funestus s.l. & $43.68 \%[31.73-55.63]$ & $62.11 \%[45.31-78.90]$ \\
\hline Kanyerere & Northern & An. funestus s.l. & $42.86 \%$ & $64.29 \%$ \\
\hline Koromije & Northern & An. gambiae s.l. & $59.42 \%[41.89-76.95]$ & $66.67 \%[38.52-94.81]$ \\
\hline
\end{tabular}

Table 5. Average 30-min knock-down and 24-h mortality to the diagnostic dose of alpha-cypermethrin (CDC bottle bioassays: $12.5 \mu \mathrm{g} / \mathrm{ml}$ ), among wild Anopheles species, collected from three study clusters in Misungwi, 2018.

the rainy season (sporozoite rates of $1.85 \%$ [95\% CI 0.23-13.24] and 3.59\% [95\% CI 1.34-9.23] during the dry and wet seasons, respectively) (Table 3 ).

In Misungwi district, malaria transmission occurs throughout the year. The average Entomological Inoculation Rate (EIR), weighted to account for the proportion of sampled Anopheles vectors processed for Plasmodium sporozoite infection, was 4.4 infective bites per house per month, approximately 53.3 per house per year, with variation in transmission intensities across the study area and seasons (Tables 2, 3). Overall, An. funestus s.s. was the major malaria vector responsible for $96.5 \%$ of total transmission (Table 3). Communities living in the southern part of the study area experienced significantly higher malaria transmission $(E I R=9.6)$ compared to the northern zone $(\mathrm{EIR}=0.6)$ (Table 2). Monthly EIR was higher in the wet compared to the dry season, for both An. funestus s.l. (3.82 vs. 2.85) and An. gambiae s.l. (3.59 vs. 1.85; Table 3).

Anopheles feeding and resting behaviours. A total of 1108 Anopheles vectors were sampled using four collection methods (CDC-LTs indoors, Furvela tent traps outdoors (Fig. 3D), Prokopack aspirators indoors and outdoors), in 96 houses across 48 clusters between December 2018 and January 2019.

As summarized in Table 4, the greatest proportions of Anopheles were sampled by indoor CDC-LTs (48.4\%) and outdoor tent traps (41.9\%). An. arabiensis and An. gambiae s.s. showed similar tendencies of feeding both indoors (54.7\% and $45.3 \%$ collected in CDC-LTs, respectively) and outdoors (51.8\% and $48.2 \%$ collected in tent traps, respectively) but An. arabiensis had a much stronger exophilic habit than An. gambiae s.s. (89.5\% [95\% CI 54.3-98.4] vs. 10.5\% [95\% CI 1.6-45.7] in Prokopack collections outdoors, respectively) (Table 4). An. funestus s.s. demonstrated similar behaviour to An. gambiae s.s., predominantly feeding indoors (CDC-LT collections) and outdoors (tent trap collections) (66.4\% and 50.2\%, respectively) and resting indoors (Prokopack collections) (48.2\% [95\% CI 23.3-73.2]). Sporozoite rates were higher in samples collected indoors (range between 1.7\% [95\% CI 0.8-3.5] and 3.9\% [95\% CI 1.0-14.4]), compared to outdoors (range between $0 \%$ and $1.7 \%$ [95\% CI 0.8-3.6]) (Table 4). Malaria transmission both indoors and outdoors was solely due to An. gambiae s.s. and An. funestus s.s.; none of the vectors collected resting outdoors were sporozoite positive.

Phenotypic resistance and underlying molecular and metabolic resistance mechanisms. Wild populations of An. funestus s.l. and An. gambiae s.l. from across the study area were confirmed resistant to the diagnostic concentration of alpha-cypermethrin, with mean 30-min knock-down ranging from 43.7 to $59.4 \%$ (Table 5). Similarly, both species were resistant to permethrin, with average 24-h mortality ranging between 38.3 and $56.5 \%$ (Table 6). In general, levels of resistance to both pyrethroids were comparable between An. gambiae s.l. and An. funestus s.l., as well as between northern and southern study zones (Fig. 2C; Tables 5, 6).

Overall, the majority $91.9 \%$ (565/615) of An. funestus s.l. mosquitoes tested in bioassays were confirmed by PCR as An. funestus s.s, with a small proportion of An. parensis $(7.8 \% ; 48 / 615)$. An. gambiae s.l. from bioassays 


\begin{tabular}{|l|l|l|l|c|}
\hline Study ward & Location & Anopheles species tested & $\mathbf{6 0}$ min Knock-down $[\mathbf{9 5 \%} \mathbf{C I}]$ & $\mathbf{2 4}$ h Mortality $[\mathbf{9 5} \% \mathbf{C I}]$ \\
\hline Bulemeji & Northern & An. gambiae s.l. & $16.67 \%[9.09-28.57]$ & $38.33 \%[24.30-52.37]$ \\
\hline Idetemya & Northern & An. gambiae s.l. & $65.22 \%[47.32-83.11]$ & $56.52 \%[35.21-75.67]$ \\
\hline Ilujamate & Southern & An. funestus s.l. & $38.00 \%[26.65-49.35]$ & $40 \%[28.39-51.61]$ \\
\hline Kanyerere & Northern & An. gambiae s.l. & $27.12 \%[13.92-40.32]$ & $38.98 \%[12.00-65.97]$ \\
\hline Mamaye & Northern & An. gambiae s.l. & $33.33 \%[19.30-47.37]$ & $45.00 \%[35.81-54.19]$ \\
\hline
\end{tabular}

Table 6. Average 60-min knock-down and 24-h mortality to the diagnostic dose of permethrin (WHO tube bioassays: 0.75\%), among wild Anopheles species, collected from five study wards in Misungwi, 2018.
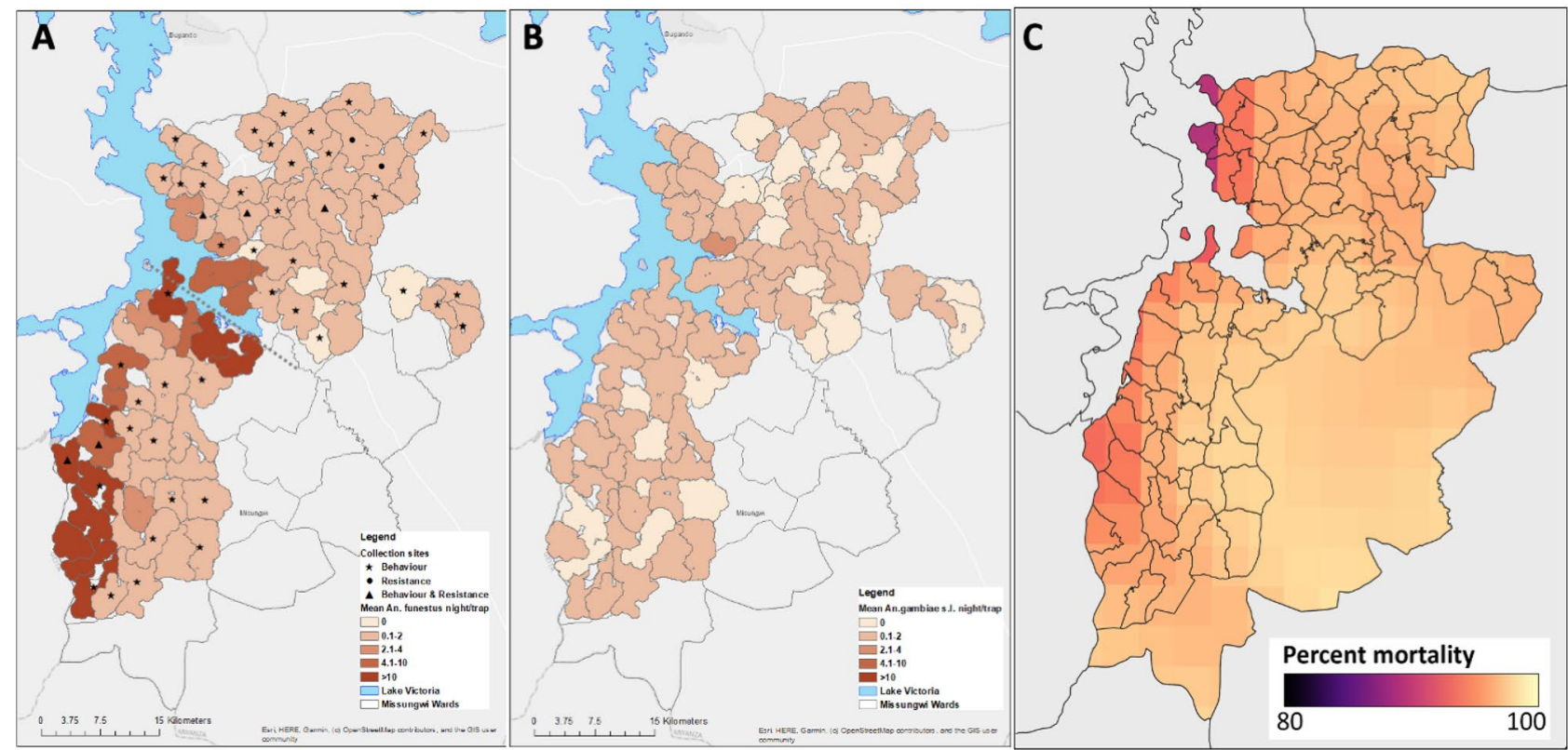

Figure 2. Study area in Misungwi district, North-West Tanzania, displaying (A) distribution of Anopheles funestus s.l. and collection methods per cluster (hashed line indicates delineation between northern and southern clusters); (B) distribution of Anopheles gambiae s.l.; and (C) predicted alpha-cypermethrin resistance for An. gambiae s.l. (mean percentage mortality). The figure was created using ArcGIS 10.8.1 (Environmental Systems Research Institute (ESRI) Redlands, California, USA, http://www.esri.com/arcgis) ${ }^{32}$.

that were tested for sibling species identification, were classified as similar proportions of An. gambiae s.s. (45.3\%; 48/106) and An. arabiensis (54.7\%; 58/106).

Three hundred and twenty-two field collected An. gambiae s.l. were screened for the presence of L1014F- $k d r$ and L1014S- $k d r$ mutations. At the population level, homozygous L1014S- $k d r$ was present in almost all An. gambiae s.s. individuals (98\%; 90/92), with evidence for significant deviations from Hardy-Weinberg equilibrium $\left(\chi^{2}=40.21 ; \mathrm{p}<0.0001\right)$. No L1014S- $k d r$ or L1014F- $k d r$ were detected in any An. arabiensis tested $(\mathrm{n}=230)$; L1014F- $k d r$ was not detected in any An. gambiae s.s. individuals.

Comparison of metabolic gene expression in An. gambiae s.s. collected from Mamaye ward (northern zone) demonstrated up-regulation of CYP6M2 (fold change; $\mathrm{FC}=0.37$ [95\% CI 0.20-0.43]), CYP6P3 (FC $=1.58$ [95\% CI 0.89-2.07]), CYP6P4 (FC=0.78 [95\% CI 0.46-1.11]) and CYP9K1 ( $\mathrm{FC}=1.58$ [95\% CI 1.19-4.80]).

Mean mortality $24 \mathrm{~h}$ after exposure to the standard diagnostic dose of alpha-cypermethrin was predicted for An. gambiae s.l. in 2017 using a geospatial model. The model used data from WHO susceptibility tests conducted from 2005 to 2017 and incorporated associations between resistance and potential explanatory variables such as ITN coverage using three different machine learning approaches. Predicted mean mortality in An. gambiae s.l. for each $5 \times 5 \mathrm{~km}$ square (Fig. 2C) was high across Tanzania in 2017. Within Misungwi, the lowest mortalities/ highest resistance occurred in the West and North-West.

\section{Discussion}

Despite substantial gains achieved in malaria control across Tanzania over the past 20 years, attributable to improved quality and access to diagnostics and treatment and the widespread scale-up of LLINs and targeted IRS, localised transmission persists, especially in the Lake Region. Study findings demonstrated that An. funestus s.l. is becoming a dominant, efficient malaria vector species in Misungwi district, North-West Tanzania in an area with high coverage of standard pyrethroid LLINs and historical IRS activities. A similar phenomenon has 

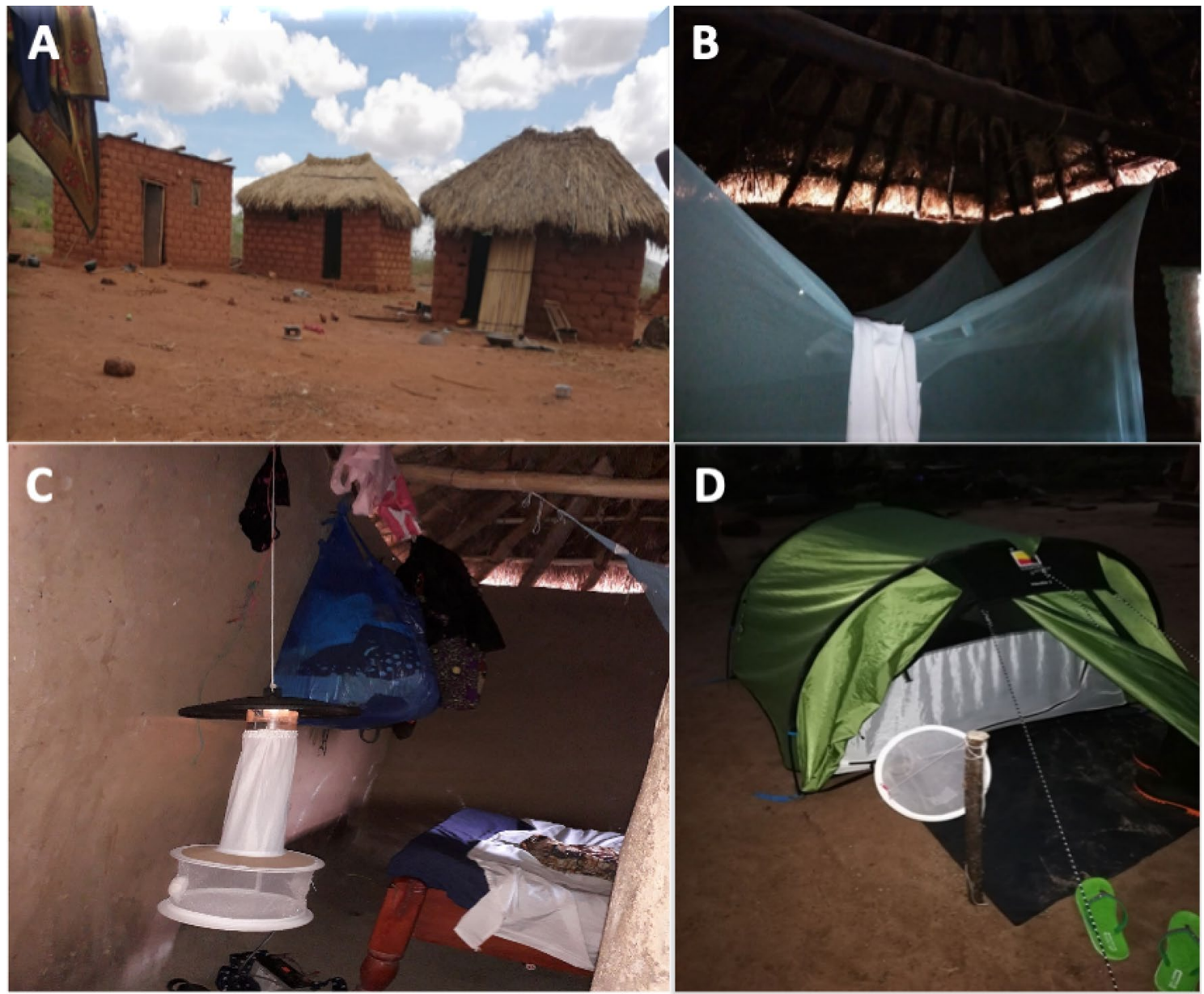

Figure 3. (A) Examples of different traditional house constructions, using local materials. (B) The inside of a typical house with open eaves. (C) A CDC-LT hung at the base of a sleeping space for sampling mosquitoes indoors. (D) A Furvela tent trap set up for catching host-seeking female Anopheles mosquitoes outdoors.

recently been reported from south-eastern Tanzania ${ }^{25}$; however, our study indicates this shift in species composition may not be restricted to the south of the country.

Around Lake Victoria, species abundance and transmission intensity vary quite considerably spatially and temporally ${ }^{28}$, with implications for the deployment of effective malaria vector control interventions. These heterogeneities likely reflect differences in climatic conditions such as rainfall and ecological settings, which support the breeding of particular vector species ${ }^{29}$. In our study, overall vector densities were significantly higher in villages located in the southern part of the study district compared to the northern clusters. An. gambiae s.s. and An. arabiensis occurred across Misungwi district, however, An. gambiae s.s. abundance was lower in the South and concentrated mostly in the North. By comparison, An. funestus s.s. was equally distributed throughout the district in sympatry with An. arabiensis and An. gambiae s.s. but found at the highest densities along shorelines and waterways feeding into Lake Victoria. The spatial variation of Anopheles sibling species may be explained by several factors linked to ecological features, including turbidity, water quality, relative humidity, temperature, vegetation type and/or socioeconomic parameters, such as ownership and usage of insecticide-based vector control measures and livestock density, as observed in previous studies conducted on the Kenyan side of Lake Victoria $^{34}$. An. gambiae s.s. are known to breed in rain-dependent temporary habitats ${ }^{35}$, while An. funestus s.s. and An. arabiensis can colonize large permanent aquatic habitats, some with large vegetation, in arid and highland $\operatorname{areas}^{36,37}$. Most residents in Misungwi district, especially in the southern clusters, traditionally stored rainwater for domestic purposes and animal husbandry in large, permanent man-made pools, locally called "Rambo", which were filled throughout the year and could serve as potential breeding sites for An. funestus s.s. and An. arabiensis, even in the dry season; the higher density of An. gambiae s.s. during the rainy season is likely due to increased availability of temporary breeding $\operatorname{sites}^{38,39}$. In addition, agricultural practices such as irrigated rice paddies create diverse aquatic mosquito breeding habitats that could influence the co-existence and abundance of different vector species in the study area ${ }^{40,41}$. An. funestus s.l. was collected in both seasons but peaked during the dry season, consistent with its ability to develop in habitats that can sustain desiccation ${ }^{42}$. Of concern, both An. gambiae s.l. and An. funestus s.l. malaria vector species were present during different seasons, favoured by distinct climatic and ecological conditions, sustaining malaria transmission throughout the region and the year. Of all mosquitoes sampled in this study, Culex species were the most abundant, contributing to $67.6 \%$ of the 
indoor host-seeking population. Previous studies in Tanzania have highlighted that Culex species, commonly referred to as "the house mosquito", predominant in malaria-endemic communities ${ }^{43}$, and when resistant to public health insecticides, can jeopardise community adherence to vector control interventions, due to perceived failure of these strategies ${ }^{44,45}$.

This study estimated that each household could receive an average of more than 53 infective bites per year from both major vector species (An. funestus s.s. and An. gambiae s.s.) despite high coverage of LLINs. Comparably high EIRs have also been reported from other rural and peri-urban regions of East Africa, including South-Central Tanzania ${ }^{46}$, coastal Kenya ${ }^{47}$ and South-West Ethiopia ${ }^{48}$. Furthermore, the annual EIR was ten times higher in villages located in the southern part of the study district compared to the North. In northern clusters, where An. gambie s.s. and An. funestus s.s. co-existed, even though An. gambiae s.s. was present in very low numbers, these two species generally had equivalent Plasmodium infection rates. Both An. gambiae s.s. and An. funestus s.s. can be highly anthropophilic and endophilic, but the former species may be more aggressive and efficient vector in terms of malaria transmission, possibly due to host competition. In the southern study clusters, malaria transmission was almost exclusively mediated by An. funestus s.s. Only a single P. falciparum-infected An. arabiensis was collected during the study which might be explained by its highly opportunistic behaviour, feeding on both animals and humans; in the absence of the latter host it can display strongly zoophilic feeding preferences for livestock, of which close to half of the households owned ${ }^{49,50}$. This species is also known for its more exophilic tendencies compared to An. gambiae s.s. ${ }^{50,51}$ and can easily adapt and feed outdoors in response to insecticidal interventions ${ }^{52}$, especially when human or animal populations are available outside ${ }^{12}$. Our indoor and outdoor collections generally support these behavioural assumptions, with both An. gambiae s.s. and An. funestus s.s. sampled in similar proportions across different traps, with the exception of An. gambiae s.s., which was found at very low densities resting outdoors. The occurrence of highly endophilic and anthropophagic vectors such as An. funestus s.s. host-seeking or resting outdoors could be linked to behavioural divergence among vector populations and/or chromosomal inversion polymorphisms $s^{53,54}$, as well as human behavioural changes ${ }^{55}$. However, in our study, more sporozoite-harbouring mosquitoes were collected in houses, suggesting ongoing malaria transmission is still occurring inside, despite high intervention coverage. The strongly exophilic behaviour of An. arabiensis indicated that LLINs and IRS in Misungwi district may have minimal effects against this species; although its relative importance in local malaria transmission appears diminished. Moreover, the presence of infected An. gambiae s.s. and An. funestus s.s. outdoors, coupled with a degree of exophagic behaviour, suggests that additional control tools targeting outdoor vector populations may warrant consideration in the study area ${ }^{56}$.

All three major vector species (An. gambiae s.s., An. arabiensis and An. funestus s.s.), displayed low levels of susceptibility to alpha-cypermethrin and permethrin, primarily due to selection pressure from prolonged use of pyrethroid-based LLINs and likely enhanced by concurrent agricultural pesticide application ${ }^{57-59}$. Because vectors for resistance monitoring were collected as adults of unknown age from house walls, and phenotypic resistance declines with age $e^{60,61}$, it is probable that our measurements of pyrethroid resistance may be an underestimation. Study findings align with others in the Lake Zone and across Tanzania, demonstrating low mortality among vector populations to the diagnostic doses of pyrethroids ${ }^{9,58,62}$. Data collected across Africa indicated that previously pyrethroid resistance was higher in East African populations of An. funestus s.l. compared to East African populations of An. gambiae s.l. up to 2017, but this difference was not detected in Misungwi in 2018-1963,64. It is noteworthy that our bioassays presented higher levels of resistance in An. gambiae s.l. in comparison to those shown in our map produced by geospatial models of phenotypic resistance to alpha-cypermethrin for the year 2017, suggesting pyrethroid resistance may be increasing in An. gambiae s.l. populations in the region. In Misungwi district, population-level frequency of the L1014S- $k d r$ mutation was practically fixed in An. gambiae s.s., while CYP6M2, CYP6P3, CYP6P4 and CYP9K1 were modestly upregulated by comparison to reports from West Africa ${ }^{65-67}$. These results indicate that both target site and metabolic mechanisms may be driving pyrethroid resistance in An. gambiae s.s. in this study area. However, further investigation is necessary to identify resistance mechanisms specific to these field populations, including those in An. funestus s.l., which were unavailable to us at the time of this study, for prospective monitoring and to improve our understanding of the specificity of resistance mechanisms to individual interventions and the likelihood of selecting for cross-resistance between active ingredients ${ }^{65-67}$. Importantly, intense insecticide resistance may partially explain the persistent malaria transmission in Misungwi district, highlighting the urgent need for novel vector control tools, containing different insecticide classes and combinations. This study was undertaken prior to the phase III evaluation of novel bi-treated LLINs containing a pyrethroid and either a pyrrole (chlorfenapyr), a synergist (piperonyl butoxide; PBO) or a juvenile growth hormone inhibitor (pyriproxyfen; PPF) ${ }^{31}$, which may have the potential to control malaria transmitted by pyrethroid-resistant vector species.

This study was conducted to characterize baseline vector population bionomics and malaria transmission dynamics in Misungwi district, with some limitations. Because mosquito collections spanned five months of the year, encompassing the short rainy season (October-December 2018) and part of the dry season (August and September 2018), vector densities, sibling species composition and sporozoite rates reported in this study may not be representative of the annual variation in this area. Additional studies are ongoing investigating indepth the association between vector spatial distributions and key ecological indices, and to identify insecticide resistance mechanisms in An. funestus s.l., which at the time of study design, was not anticipated to emerge as the major vector species in Misungwi district.

\section{Conclusion}

In Misungwi district, North-West Tanzania, An. funestus s.s. is the leading malaria vector species, predominating in southern villages of the study site, across dry and wet seasons. An. gambiae s.s. was present in much lower densities, concentrated mostly in the North during the wet season, potential driving malaria epidemics. Annual 
EIR was high, despite high LLIN usage, but variable within a small geographical area, influenced by vector species diversity and bionomics, with serious epidemiological implications for malaria control. An. gambiae s.s., An. arabiensis and An. funestus s.s. were found similarly resistant to pyrethroids, with high frequencies of target site alleles and overexpression of detoxification genes identified in An. gambiae s.s. Study findings highlight the urgent need for novel vector controls strategies, which incorporate new chemical classes, to control malaria transmitted by pyrethroid-resistant vector populations and sustain gains in malaria control across the Lake Region.

\section{Methods}

Study area characteristics. The study was carried in Misungwi district (latitude $2.85000 \mathrm{~S}$, longitude 33.08333 E) in North-West Tanzania on the southern shore of Lake Victoria (Fig. 1A). Misungwi lies at an altitude of $1150 \mathrm{~m}$ above sea level, with a population of approximately 351,607 according to the National population and housing census of $2012^{68}$. The district experiences a dry season typically between June and September and two annual rainy seasons; the long-rainy season between February and May and a short-rainy season between November and December with average annual rainfall ranging between 0.5 and $58.8 \mathrm{~mm}$. The district is geographically divided into two main agro-ecological zones (northern and southern zones), based on the vegetation land cover and amount of rainfall. The local communities practice rice, millet and cotton farming, domestic animal rearing, fishing and have small-scale businesses as sources of income and food. In preparation for the RCT, the study area was sub-divided into 86 clusters, containing 72 villages made up of 453 hamlets from 17 wards (Fig. 2). Detailed criteria and methodology used for cluster formation is described elsewhere ${ }^{31}$.

Across Misungwi district, a typical compound was comprised of a house and cattle shed. Houses were generally constructed from both modern and traditional materials and most houses had eave spaces (an opening between the wall and the roof for ventilation) (Fig. 3A,B). The area experiences moderate to high malaria transmission and malaria incidence peaks shortly after the rainy seasons ${ }^{69}$. Recent studies conducted in 2010 and 2017 reported a malaria prevalence of $51.3 \%$ across all age groups and $46.3 \%$ in school children (7-14 years). LLINs mainly Olyset and PermaNet 2.0 obtained through national bed net distribution campaigns have been the primary malaria control method in the study area ${ }^{4,70}$ and IRS was last conducted in this region in 2015. A preliminary survey carried out by our study team in 2018 found An. gambiae s.s. An. arabiensis and An. funestus s.s. as the predominant malaria vector species in the study area.

Environmental characteristics of northern and southern clusters. To characterize the study area with regards to climatic and environmental conditions, high spatial resolution satellite remote sensing and other geospatial data were downloaded in raster (i.e. gridded) format from publicly available data sources and processed using ArcGIS 10.5.1 (ESRI, Redlands, USA). Data on eight bioclimatic variables at $1 \mathrm{~km}^{2}$ spatial resolution, representing averages for the years 1970-2000, were obtained from the WorldClim 2 database ${ }^{71}$ : annual mean temperature (Bio1), temperature seasonality (Bio4), maximum and minimum temperature of the warmest month (Bio 5 and 6), annual precipitation (Bio12), precipitation of the wettest and driest months (Bio13 and 14 ), and precipitation seasonality (Bio15). Global elevation data were obtained for the study area from NASA's Shuttle Radar Topography Mission (SRTM) 4.1 at 90-m spatial resolution ${ }^{72}$. Global landcover data were obtained from the European Space Agency GlobCover 2009 project, available at 300-m spatial resolution (C ESA 2010 and UCLouvain; http://due.esrin.esa.int/page_globcover.php). These data identify 22 landcover types, of which 12 were identified in the study area. Zonal mean statistics for the northern and southern clusters were calculated for each bioclimatic variable and elevation using the spatial analyst toolbox in ArcGIS; cluster means were then averaged for each zone (Supplementary Table S1). The proportion of cells within each of the northern and southern zones that were classified as different landcover types were similarly calculated; Supplementary Table S1 shows the results for the five dominant landcover types that were present in the study area [mosaic vegetation (i.e. grassland/shrubland/forest: 50-70\%/cropland: 20-50\%), herbaceous vegetation (i.e. grassland/savannas), shrubland, broadleaf deciduous forest/woodland, and grassland or woody vegetation on regularly flooded or waterlogged soil], which represent $98.9 \%$ and $97.5 \%$ of the total area of the northern and southern clusters, respectively. While the available data sources, and hence these estimates, are derived from time periods prior to the study period, we assume that the estimates accurately reflect relative differences in climatic and environmental conditions across the study area.

Indoor and outdoor entomological surveillance. Two cross-sectional entomological field surveys were conducted between August and December 2018 in all 86 clusters, using CDC-LTs (John W Hock Company, USA). Eight households were randomly selected from a census list of households generated during baseline enumeration. CDC-LTs were hung next to the feet of an occupant sleeping under an ITN/untreated net (about $1 \mathrm{~m}$ from the ground), between 19:00 and 7:00. A questionnaire was administered to the head of the household to gather information related to the house structure (type of wall and roofing materials, window screening, number of rooms, number of sleeping places, presence of eaves), and coverage and usage of LLINs/untreated nets. Direct observation was also used during data collection to validate participant answers.

Assessment of Anopheles vector feeding and resting behaviours indoors and outdoors was undertaken in 48 clusters between December 2018 and January 2019. Two households per cluster were randomly selected, and each house was installed with a CDC-LT indoors and an occupied Furvela tent trap outdoors ${ }^{73}$. Both Furvela and CDC-LTs were switched on at 19:00 and off at 7:00. Indoor and outdoor resting adult Anopheles were collected from the same houses using a 12 voltage battery-powered Prokopack aspirator ${ }^{74}$, and manual mouth aspirators ${ }^{75,76}$. Systematic sampling of adult resting mosquitoes on the walls, roofs and floors were conducted for up to three minutes depending on the size of the room. Outdoor collections were performed from potential resting sites around the house, such as open resting structures, cow sheds and pit latrines. 
Insecticide resistance testing. Insecticide resistance profiles of wild populations of An. gambiae s.l. and An. funestus s.l. were assessed in six clusters selected on the basis of high Anopheles population densities. Due to difficulties locating reliable, productive breeding sites for larval sampling, particularly for An. funestus s.l., instead adult female Anopheles resting indoors were collected using both Prokopack and mouth aspirators for resistance testing ${ }^{74}$. Mosquitoes were separated by species complex and supplied with $10 \%$ glucose solution for $72 \mathrm{~h}$ to allow digestion of blood meal, prior to bioassay testing with permethrin and alpha-cypermethrin. These two pyrethroids were selected on the basis that they are the partner insecticides in next-generation LLINs (Olyset Plus, Royal Guard and Interceptor G2), which were about to be distributed across the study district ${ }^{77}$. In WHO tube assays, 20-25 gravid female An. gambiae s.l. or An. funestus s.l. of unknown age were exposed to $0.75 \%$ permethrin for $60 \mathrm{~min}^{78}$. In CDC bottle bioassays, 20-25 gravid female An. gambiae s.l. or An. funestus s.l. of unknown age were exposed to $12.5 \mu \mathrm{g} / \mathrm{ml}$ alpha-cypermethrin for $30 \mathrm{~min}^{79}$. For both assays, knock-down was recorded at the end of the diagnostic exposure time (30 or $60 \mathrm{~min}$ after exposure, for CDC or WHO bioassays, respectively), and final mortality was scored after $24 \mathrm{~h}$. All mosquitoes tested in bioassays were stored individually for sibling species identification.

Mosquito processing, species identification and sporozoite detection. Adult female mosquitoes collected from the cross-sectional surveys, resistance bioassays and behaviour study were sorted and identified based on their morphology, separating An. gambiae s.l. from An. funestus s.l. and from other genera according to Gillies and Coetzee ${ }^{80}$. At least three female An. gambiae s.l. and three An. funestus s.l. per household/ per collection method was analysed for presence of Plasmodium falciparum CSP using enzyme-linked immunosorbent assay (CSP-ELISA) ${ }^{81}$. All CSP positive samples and a sub-sample of CSP negative An. gambiae s.l. and An. funestus s.l. from the cross-sectional surveys, resistance bioassays and behaviour study, were randomly picked per house and tested for species identification. DNA was extracted from legs/wings and TaqMan PCR assays were performed to distinguish sibling species in An. gambiae ${ }^{82}$ or An. funestus complexes ${ }^{83}$.

Identification of insecticide resistance mechanisms. A subsample of An. gambiae s.s. and An. arabiensis were genotyped for L1014F- $k d r$ and L1014S- $k d r$ mutations associated with pyrethroid and DDT resistance, using TaqMan PCR assays ${ }^{84}$. Blood-fed indoor resting female adult mosquitoes (F0s) were collected using mouth aspirators from three wards, sampled for phenotypic resistance testing. Mosquitoes were held for 3-4 days to allow for blood meal digestion. Individual An. gambiae s.l. were placed into Eppendorf tubes containing moist filter papers and forced to lay eggs, as previously described ${ }^{23}$. The first 3-5 day emerged F1 adults from each parent were stored individually in RNAlater and preserved at $-20{ }^{\circ} \mathrm{C}$ for gene expression analysis. Expression profiles for metabolic detoxification genes in a sub-sample of 250-300 F1 wild-caught female An. gambiae s.s. mosquitoes were determined using quantitative reverse transcriptase PCR (qRT-PCR) ${ }^{85,86}$. Individual mosquitoes were first tested for species identification, and only mosquitoes identified as An. gambiae s.s. were analyzed. A minimum of 5 pools of 10 An. gambiae s.s. were analysed for CYP6M2, CYP6P3, CYP6P4 and CYP9K1 gene expression $^{85}$.

Data analysis. Field data were entered into an Open Data Kit (ODK) form. Data analysis was performed using Stata/IC 15.1 (Stata Corp., College Station, USA) ${ }^{87}$. Mean Anopheles caught per night per household, sporozoite rate and their 95\% confidence intervals (CIs) were estimated according to study zones (North or South), season (wet or dry) and Anopheles species. Anopheles vector population density and EIR were analysed and compared between study zones and seasons using multilevel negative binomial regression taking into account clustering effect. The EIR was calculated at household level as the average number of CSP-ELISA positive mosquitoes per night and was weighted to account for the proportion of collected Anopheles processed for CSPELISA. Logistic regression was used to compare sporozoite rates between the two study zones and seasons. The proportion of households with at least one LLIN was computed by dividing total nets observed and recorded by total households surveyed. Net access was estimated from the proportion of households with enough LLINs over total sleeping places. Unimproved houses were classified as houses with open eaves, unscreened windows, and were constructed from traditional low-quality materials such as a thatched roof, mud and non-plastered walls. Improved houses had closed eaves, with mosquito proof mesh on the windows, and were built with improved modern materials such as an iron sheet as a roof, brick/blocks, with plastered walls.

For resistance phenotyping, mean percentage knock-down/mortality post-exposure was calculated and interpreted following the WHO and CDC criteria ${ }^{78,79}$. Susceptibility thresholds were considered at the diagnostic time ( $24 \mathrm{~h}$ and $30 \mathrm{~min}$ post-exposure for WHO and CDC bioassays, respectively). Mean mosquito mortality between 98 and 100\% indicated full insecticide susceptibility, 90-97\% showed suspected resistance that needed confirmation and less than $90 \%$ indicated confirmed resistance ${ }^{78,79}$. When control mortality was between 5 and $20 \%$, results were corrected using Abbott's formula ${ }^{78}$. If the control mortality was $\geq 20 \%$, the test was discarded ${ }^{78}$.

Gene expression and fold-change, relative to the susceptible laboratory strain An. gambiae s.s. Kisumu were calculated according to the $2-\Delta \Delta \mathrm{Cq}$ method $^{88}$ after standardisation with housekeeping genes (elongation factor; $E F$ and 40 S ribosomal protein $\mathrm{S} 7$; RPS7).

To estimate spatial and temporal trends in pyrethroid resistance using the available field data, which are sparse and have a heterogeneous distribution, a total of 6423 observations of mortality from WHO susceptibility tests from 2005 to 2017 were used to inform a Bayesian geostatistical ensemble model. The model was also informed by a suite of 111 explanatory variables describing potential drivers of selection for resistance such as ITN coverage and produced estimates of mean mortality in An. gambiae s.l. for two regions of Africa ${ }^{64}$. Here we present the results for alpha-cypermethrin resistance in Misungwi in 2017. 
Ethical approval and consent to participate. This study is part of an ongoing RCT in Misungwi (clinical trial registration: NCT03554616) which obtained ethical clearance from the National Institute for Medical Research (NIMR), Tanzania (NIMR/HQ/R.8a/Vol. IX/2743) and the London School of Tropical Medicine and Hygiene, United Kingdom (LSHTM ethics ref: 14952) ${ }^{31}$. All study procedures were performed in accordance with relevant guidelines and regulations. Prior to study initiation, community consent was sought from village leaders and written, informed consent was obtained from the heads of all households selected for participation. Study information, including the study purpose, risks and benefits, was provided to participants in Swahili.

\section{Data availability}

The data sets generated and/or analysed during the current study are not public but are available from the corresponding author on reasonable request.

Received: 2 April 2021; Accepted: 8 June 2021

Published online: 29 June 2021

\section{References}

1. Bhatt, S. et al. The effect of malaria control on Plasmodium falciparum in Africa between 2000 and 2015. Nature 526(7572), 207-211 (2015).

2. WHO. World Malaria Report (WHO, 2020).

3. WH Organization. High Burden to High Impact: A Targeted Malaria Response (World Health Organization, 2018).

4. Ministry of Health CD, Gender, Elderly, Children MoH, National Bureau of Statistics, Office of the Chief Government Statistician, ICF. Tanzania Malaria Indicator Survey 2018. MoHCDGEC, MoH, NBS, OCGS, and ICF Dar es Salaam, Tanzania, and Rockville; 2018.

5. Yukich, J. et al. Sustaining LLIN coverage with continuous distribution: The school net programme in Tanzania. Malar. J. 19, 1-12 (2020).

6. PMI. Presidents Malaria Initiative, Malaria Operational Plan: Tanzania FY 2019. USAID. 2019.

7. Ranson, H. \& Lissenden, N. Insecticide resistance in African Anopheles mosquitoes: A worsening situation that needs urgent action to maintain malaria control. Trends Parasitol. 32(3), 187-196 (2016).

8. Kisinza, W. N. et al. Multiple insecticide resistance in Anopheles gambiae from Tanzania: A major concern for malaria vector control. Malar. J. 16(1), 439. https://doi.org/10.1186/s12936-017-2087-2 (2017).

9. Matiya, D. J., Philbert, A. B., Kidima, W. \& Matowo, J. J. Dynamics and monitoring of insecticide resistance in malaria vectors across mainland Tanzania from 1997 to 2017: A systematic review. Malar. J. 18(1), 102. https://doi.org/10.1186/s12936-019-2738-6 (2019).

10. Moiroux, N. et al. Changes in Anopheles funestus biting behavior following universal coverage of long-lasting insecticidal nets in Benin. J. Infect. Dis. 206(10), 1622-1629 (2012).

11. Reddy, M. R. et al. Outdoor host seeking behaviour of Anopheles gambiae mosquitoes following initiation of malaria vector control on Bioko Island, Equatorial Guinea. Malar. J. 10(1), 184. https://doi.org/10.1186/1475-2875-10-184 (2011).

12. Russell, T. L. et al. Increased proportions of outdoor feeding among residual malaria vector populations following increased use of insecticide-treated nets in rural Tanzania. Malar. J. 10(1), 80 (2011).

13. McCann, R. S. et al. Reemergence of Anopheles funestus as a vector of Plasmodium falciparum in western Kenya after long-term implementation of insecticide-treated bed nets. Am. J. Trop. Med. Hyg. 90(4), 597-604 (2014).

14. Coetzee, M. \& Koekemoer, L. L. Molecular systematics and insecticide resistance in the major African malaria vector Anopheles funestus. Annu. Rev. Entomol. 58, 393-412 (2013).

15. Coetzee, M. \& Fontenille, D. Advances in the study of Anopheles funestus, a major vector of malaria in Africa. Insect Biochem. Mol. Biol. 34(7), 599-605 (2004).

16. Gillies, M. T., \& De Meillon, B. The Anophelinae of Africa south of the Sahara (Ethiopian Zoogeographical Region). 1968.

17. Cohuet, A. et al. High malaria transmission intensity due to Anopheles funestus (Diptera: Culicidae) in a village of savannah-forest transition area in Cameroon. J. Med. Entomol. 41(5), 901-905 (2004).

18. Muturi, E. J. et al. Spatial distribution, blood feeding pattern, and role of Anopheles funestus complex in malaria transmission in central Kenya. Parasitol. Res. 105(4), 1041-1046 (2009).

19. Riveron, J. M. et al. Escalation of pyrethroid resistance in the malaria vector Anopheles funestus induces a loss of efficacy of piperonyl butoxide-based insecticide-treated nets in Mozambique. J. Infect. Dis. 220(3), 467-475 (2019).

20. Djouaka, R. et al. Multiple insecticide resistance in an infected population of the malaria vector Anopheles funestus in Benin. Parasit. Vectors 9(1), 1-12 (2016).

21. Riveron, J. M. et al. Multiple insecticide resistance in the major malaria vector Anopheles funestus in southern Ghana: Implications for malaria control. Parasit. Vectors 9(1), 1-9 (2016).

22. Menze, B. D. et al. Multiple insecticide resistance in the malaria vector Anopheles funestus from Northern Cameroon is mediated by metabolic resistance alongside potential target site insensitivity mutations. PLoS One 11(10), e0163261 (2016).

23. Morgan, J. C., Irving, H., Okedi, L. M., Steven, A. \& Wondji, C. S. Pyrethroid resistance in an Anopheles funestus population from Uganda. PLoS One 5(7), e11872 (2010).

24. Cuamba, N., Morgan, J. C., Irving, H., Steven, A. \& Wondji, C. S. High level of pyrethroid resistance in an Anopheles funestus population of the Chokwe District in Mozambique. PLoS One 5(6), e11010 (2010).

25. Kaindoa, E. W. et al. Interventions that effectively target Anopheles funestus mosquitoes could significantly improve control of persistent malaria transmission in south-eastern Tanzania. PLoS One 12(5), 0e177807 (2017).

26. Limwagu, A. J. et al. Using a miniaturized double-net trap (DN-Mini) to assess relationships between indoor-outdoor biting preferences and physiological ages of two malaria vectors, Anopheles arabiensis and Anopheles funestus. Malar. J. 18(1), 1-15 (2019).

27. Lwetoijera, D. W. et al. Increasing role of Anopheles funestus and Anopheles arabiensis in malaria transmission in the Kilombero Valley, Tanzania. Malar. J. 13(1), 331 (2014).

28. Kakilla, C. et al. Malaria vector species composition and entomological indices following indoor residual spraying in regions bordering Lake Victoria, Tanzania. Malar. J. 19(1), 1-14 (2020).

29. Nambunga, I. H. et al. Aquatic habitats of the malaria vector Anopheles funestus in rural south-eastern Tanzania. Malar. J. 19(1), $1-11(2020)$.

30. Thawer, S. G. et al. Sub-national stratification of malaria risk in mainland Tanzania: A simplified assembly of survey and routine data. Malar. J. 19, 1-12 (2020).

31. Mosha, J. F. et al. Protocol for a four parallel-arm, single-blind, cluster-randomised trial to assess the effectiveness of three types of dual active ingredient treated nets compared to pyrethroid-only long-lasting insecticidal nets to prevent malaria transmitted by pyrethroid insecticide-resistant vector mosquitoes in Tanzania. BMJ Open 11(3), 046664 (2021). 
32. ArcGIS E. 8.3 of ArcMap, ArcCatalog, ArcToolbox and Spatial Analyst User’s Guide. (Redlands, CA, 2002).

33. Eze, I. C., Kramer, K., Msengwa, A., Mandike, R. \& Lengeler, C. Mass distribution of free insecticide-treated nets do not interfere with continuous net distribution in Tanzania. Malar. J. 13(1), 1-10 (2014).

34. Minakawa, N., Dida, G. O., Sonye, G. O., Futami, K. \& Njenga, S. M. Malaria vectors in Lake Victoria and adjacent habitats in western Kenya. PLoS One 7(3), e32725 (2012).

35. Sinka, M. E. et al. A global map of dominant malaria vectors. Parasit. Vectors 5(1), 1-11 (2012).

36. Kelly-Hope, L. A., Hemingway, J. \& McKenzie, F. E. Environmental factors associated with the malaria vectors Anopheles gambiae and Anopheles funestus in Kenya. Malar. J. 8(1), 268 (2009).

37. Wiebe, A. et al. Geographical distributions of African malaria vector sibling species and evidence for insecticide resistance. Malar. J. 16(1), 85 (2017).

38. Adja, A. et al. Contribution of Anopheles funestus, An. gambiae and An. nili (Diptera: Culicidae) to the perennial malaria transmission in the southern and western forest areas of Côte d'Ivoire. Ann. Trop. Med. Parasitol. 105(1), 13-24 (2011).

39. Koudou, B. et al. Effects of seasonality and irrigation on malaria transmission in two villages in Côte d'Ivoire. Ann. Trop. Med. Parasitol. 104(2), 109-121 (2010).

40. Mutero, C. M. et al. A transdisciplinary perspective on the links between malaria and agroecosystems in Kenya. Acta Trop. 89(2), 171-186 (2004).

41. Wondwosen, B. et al. Rice volatiles lure gravid malaria mosquitoes, Anopheles arabiensis. Sci. Rep. 6(1), 37930. https://doi.org/10. 1038/srep37930 (2016).

42. Charlwood, J., Vij, R. \& Billingsley, P. Dry season refugia of malaria-transmitting mosquitoes in a dry savannah zone of east Africa. Am. J. Trop. Med. Hyg. 62(6), 726-732 (2000).

43. Matowo, N. S. et al. Fine-scale spatial and temporal variations in insecticide resistance in Culex pipiens complex mosquitoes in rural south-eastern Tanzania. Parasit. Vectors 12(1), 1-13 (2019).

44. Fuseini, G. et al. Increased biting rate of insecticide-resistant culex mosquitoes and community adherence to IRS for malaria control in urban malabo, bioko island, equatorial guinea. J. Med. Entomol. 56(4), 1071-1077 (2019).

45. Toé, L. P. et al. Decreased motivation in the use of insecticide-treated nets in a malaria endemic area in Burkina Faso. Malar. J. 8(1), 1-9 (2009).

46. Drakeley, C. et al. An estimation of the entomological inoculation rate for Ifakara: A semi-urban area in a region of intense malaria transmission in Tanzania. Trop. Med. Int. Health 8(9), 767-774 (2003).

47. Mbogo, C. N. et al. Relationships between Plasmodium falciparum transmission by vector populations and the incidence of severe disease at nine sites on the Kenyan coast. Am. J. Trop. Med. Hyg. 52(3), 201-206 (1995).

48. Abraham, M., Massebo, F. \& Lindtjørn, B. High entomological inoculation rate of malaria vectors in area of high coverage of interventions in southwest Ethiopia: Implication for residual malaria transmission. Parasit. Epidemiol. Control 2(2), 61-69 (2017).

49. Takken, W. \& Verhulst, N. O. Host preferences of blood-feeding mosquitoes. Annu. Rev. Entomol. 58, 433-453 (2013).

50. Mahande, A., Mosha, F., Mahande, J. \& Kweka, E. Feeding and resting behaviour of malaria vector, Anopheles arabiensis with reference to zooprophylaxis. Malar. J. 6(1), 100 (2007).

51. White, G., Magayuka, S. A. \& Boreham, P. Comparative studies on sibling species of the Anopheles gambiae Giles complex (Dipt., Culicidae): Bionomics and vectorial activity of species A and species B at Segera, Tanzania. Bull. Entomol. Res. 62(2), 295-317 (1972).

52. Kitau, J. et al. Species shifts in the Anopheles gambiae complex: Do LLINs successfully control Anopheles arabiensis?. PLoS One 7(3), e31481 (2012).

53. Guelbeogo, W. M., Sagnon, N. F., Liu, F., Besansky, N. J. \& Costantini, C. Behavioural divergence of sympatric Anopheles funestus populations in Burkina Faso. Malar. J. 13(1), 65 (2014).

54. Coluzzi, M., Sabatini, A., Petrarca, V. \& Di Deco, M. A. Behavioural divergences between mosquitoes with different inversion karyotypes in polymorphic populations of the Anopheles gambiae complex. Nature 266(5605), 832-833 (1977).

55. Finda, M. F. et al. Linking human behaviours and malaria vector biting risk in south-eastern Tanzania. PLoS One 14(6), 0217414 (2019).

56. Killeen, G. F. et al. Developing an expanded vector control toolbox for malaria elimination. BMJ Glob. Health 2, 2 (2017).

57. Reid, M. C. \& McKenzie, F. E. The contribution of agricultural insecticide use to increasing insecticide resistance in African malaria vectors. Malar. J. 15(1), 107 (2016).

58. Philbert, A., Lyantagaye, S. L. \& Nkwengulila, G. Farmers' pesticide usage practices in the malaria endemic region of North-Western Tanzania: Implications to the control of malaria vectors. BMC Public Health 19(1), 1456 (2019).

59. Matowo, N. S. et al. Patterns of pesticide usage in agriculture in rural Tanzania call for integrating agricultural and public health practices in managing insecticide-resistance in malaria vectors. Malar. J. 19(1), 257. https://doi.org/10.1186/s12936-020-03331-4 (2020).

60. Jones, C. M. et al. Aging partially restores the efficacy of malaria vector control in insecticide-resistant populations of Anopheles gambiae sl. from Burkina Faso. Malar. J. 11(1), 1-11 (2012).

61. Glunt, K. D., Thomas, M. B. \& Read, A. F. The effects of age, exposure history and malaria infection on the susceptibility of Anopheles mosquitoes to low concentrations of pyrethroid. PLoS One 6(9), e24968 (2011).

62. Protopopoff, N. et al. High level of resistance in the mosquito Anopheles gambiae to pyrethroid insecticides and reduced susceptibility to bendiocarb in north-western Tanzania. Malar. J. 12(1), 149 (2013).

63. Moyes, C. L. et al. Analysis-ready datasets for insecticide resistance phenotype and genotype frequency in African malaria vectors. Sci. Data 6(1), 1-11 (2019)

64. Hancock, P. A. et al. Mapping trends in insecticide resistance phenotypes in African malaria vectors. PLoS Biol. 18(6), e3000633 (2020).

65. Oumbouke, W. A. et al. Fine scale spatial investigation of multiple insecticide resistance and underlying target-site and metabolic mechanisms in Anopheles gambiae in central Côte d'Ivoire. Sci. Rep. 10(1), 1-13 (2020).

66. Vontas, J. et al. Rapid selection of a pyrethroid metabolic enzyme CYP9K1 by operational malaria control activities. Proc. Natl. Acad. Sci. 115(18), 4619-4624 (2018).

67. Ngufor, C. et al. Insecticide resistance profile of Anopheles gambiae from a phase II field station in Cové, southern Benin: Implications for the evaluation of novel vector control products. Malar. J. 14(1), 1-10 (2015).

68. National Bureau of Statistics. 2012 population and housing census. (Dar es Salaam, Tanzania, 2013).

69. Mosha, J. F. et al. The independent effect of living in malaria hotspots on future malaria infection: An observational study from Misungwi, Tanzania. Malar. J. 13(1), 445. https://doi.org/10.1186/1475-2875-13-445 (2014).

70. Renggli, S. et al. Design, implementation and evaluation of a national campaign to deliver 18 million free long-lasting insecticidal nets to uncovered sleeping spaces in Tanzania. Malar J. 12(1), 85 (2013).

71. Fick, S. E. \& Hijmans, R. J. WorldClim 2: New 1-km spatial resolution climate surfaces for global land areas. Int. J. Climatol. 37(12), 4302-4315 (2017).

72. Reuter, H. I., Nelson, A. \& Jarvis, A. An evaluation of void-filling interpolation methods for SRTM data. Int. J. Geogr. Inf. Sci. 21(9), 983-1008 (2007).

73. Charlwood, J. D., Rowland, M., Protopopoff, N. \& Le Clair, C. The Furvela tent-trap Mk 1.1 for the collection of outdoor biting mosquitoes. PeerJ 5, e3848 (2017). 
74. Vazquez-Prokopec, G. M., Galvin, W. A., Kelly, R. \& Kitron, U. A new, cost-effective, battery-powered aspirator for adult mosquito collections. J. Med. Entomol. 46(6), 1256-1259 (2009).

75. Service, M. Entomological Field Techniques for Malaria Control: Part I. Learner's Guide \& Part II. Tutor's Guide. Geneva: World Health Organization, 1992. Part I, 77pp, Part II, 54pp. Price Part I, Sw. fr 15; Part II, SW. fr. 12;(Developing Countries Sw. fr. 10.50 \& 8.40 Respectively). ISBN Part I, 92-4-154439-2; Part II, 92-4-15440-6 (Royal Society of Tropical Medicine and Hygiene, 1993).

76. Silver, J. B. Mosquito Ecology: Field Sampling Methods (Springer, 2007).

77. Mosha, J. F. et al. Protocol for a four parallel-arm, single-blind, cluster-randomized trial to assess the effectiveness of three types of dual active ingredient treated nets compared to pyrethroid-only long-lasting insecticidal nets to prevent malaria transmitted by pyrethroid insecticide-resistant vector mosquitoes in Tanzania. BMJ Open 11(3), e046664 (2020).

78. WHO. Test Procedures for Insecticide Resistance Monitoring in Malaria Vector Mosquitoes (World Health Organisation, 2016).

79. Brogdon, W. \& Chan, A. Guideline for Evaluating Insecticide Resistance in Vectors Using The CDC Bottle Bioassay (CDC Atlanta, 2010).

80. De GilliesMeillon, M. D. The Anophelinae of Africa South of the Sahara. Publ. South Afr. Inst. Med. Res. 54, 343 (1968).

81. Burkot, T., Williams, J. \& Schneider, I. Identification of Plasmodium falciparum-infected mosquitoes by a double antibody enzymelinked immunosorbent assay. Am. J. Trop. Med. Hyg. 33(5), 783-788 (1984).

82. Bass, C., Williamson, M. S. \& Field, L. M. Development of a multiplex real-time PCR assay for identification of members of the Anopheles gambiae species complex. Acta Trop. 107(1), 50-53 (2008).

83. Vezenegho, S. B. et al. Development of multiplex real-time PCR assays for identification of members of the Anopheles funestus species group. Malar. J. 8(1), 1-9 (2009).

84. Bass, C. et al. Detection of knockdown resistance (kdr) mutations in Anopheles gambiae: A comparison of two new high-throughput assays with existing methods. Malar. J. 6(1), 1-14 (2007).

85. Müller, P. et al. Field-caught permethrin-resistant Anopheles gambiae overexpress CYP6P3, a P450 that metabolises pyrethroids. PLoS Genet. 4(11), e1000286 (2008).

86. Mavridis, K. et al. Rapid multiplex gene expression assays for monitoring metabolic resistance in the major malaria vector Anopheles gambiae. Parasit. Vectors 12(1), 9 (2019).

87. L StataCorp. StataCorp Stata Statistical Software: Release 15.1 (StataCorp LP, 2017).

88. Rao, X., Huang, X., Zhou, Z. \& Lin, X. An improvement of the 2(-delta delta CT) method for quantitative real-time polymerase chain reaction data analysis. Biostat. Bioinform. Biomath. 3(3), 71-85 (2013).

\section{Acknowledgements}

We thank the entomology technicians for their support in the field work and Misungwi District residents for their acceptance and cooperation throughout the study. This study was funded by the Department for International Development, the UK Medical Research Council, the Wellcome Trust and the Department of Health and Social Care (\#MR/R006040/1).

\section{Author contributions}

N.P., F.W.M., M.R., M.A.K., J.F.M. and A.M. wrote the main study protocol and design the study. N.S.M., N.P., L.A.M., P.H., C.M. performed data analysis. G.I., B.S. and R.K. performed the molecular analysis. J.M., E.L. and N.S.M. supervised the study data collections. N.S.M. wrote the initial draft of the manuscript, which was revised by N.P. and L.A.M. All authors read and approved the final manuscript.

\section{Competing interests}

The authors declare no competing interests.

\section{Additional information}

Supplementary Information The online version contains supplementary material available at https://doi.org/ 10.1038/s41598-021-92741-8.

Correspondence and requests for materials should be addressed to N.S.M. or L.A.M.

Reprints and permissions information is available at www.nature.com/reprints.

Publisher's note Springer Nature remains neutral with regard to jurisdictional claims in published maps and institutional affiliations.

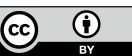

Open Access This article is licensed under a Creative Commons Attribution 4.0 International License, which permits use, sharing, adaptation, distribution and reproduction in any medium or format, as long as you give appropriate credit to the original author(s) and the source, provide a link to the Creative Commons licence, and indicate if changes were made. The images or other third party material in this article are included in the article's Creative Commons licence, unless indicated otherwise in a credit line to the material. If material is not included in the article's Creative Commons licence and your intended use is not permitted by statutory regulation or exceeds the permitted use, you will need to obtain permission directly from the copyright holder. To view a copy of this licence, visit http://creativecommons.org/licenses/by/4.0/.

(C) The Author(s) 2021 